\title{
Cytokeratin 7-negative and GATA binding protein 3-negative breast cancers: Clinicopathological features and prognostic significance
}

\author{
Shaolei Lu*iD, Evgeny Yakirevich, Li Juan Wang, Murray B. Resnick and Yihong Wang
}

\begin{abstract}
Background: Cytokeratin 7 (CK7) and GATA binding protein 3 (GATA3) are considered as immunohistochemical hallmarks of breast cancers; however, there are breast tumors lacking these markers. Clinicopathological characterization of CK7 negative breast cancer has not been addressed previously and similar studies on GATA3 negative tumors are limited.

Methods: This study included 196 consecutive cases of Nottingham Grade 3 breast cancers with 159 cases of Grade 1 and Grade 2 tumors for comparison. CK7 and GATA3 expression was correlated with patient's age, histological type, pathological grade and stage, hormone receptor status, molecular subtype and overall survival.

Results: CK7 negativity was seen in 13\% of Grade 3, 9\% of Grade 2, and 2\% of Grade 1 cases $(P=0.0457)$. Similarly, $28 \%$ of Grade 3, 5\% of Grade 2 and 2\% of Grade 1 cases were GATA3 negative $(P<0.0001)$. CK7 negative tumors did not show association with other clinicopathological parameters. GATA3 negative tumors were enriched in the basal-like molecular subgroup and were associated with negative estrogen receptor (ER) and negative progesterone receptor (PR) statuses. Both CK7 and GATA3 expression showed no association with overall survival in patients with Grade 3 tumor.

Conclusions: This is the first study to characterize CK7 negative breast tumors in the context of clinicopathology. Profiling the CK7 negative and GATA3 negative breast cancers helps to understand the biology of these specific tumor subgroups and may aid in their diagnosis.
\end{abstract}

Keywords: CK7, GATA3, Breast carcinoma, Immunohistochemistry

\section{Background}

Breast cancer is the most common cancer in females and is the second leading cause of cancer death in females after lung cancer [1]. It is estimated that in 2018 $30 \%$ of newly diagnosed malignancies in females in the United States will be breast cancer [1]. Most of the primary breast cancers are initially diagnosed by breast biopsy following imaging studies. Cytokeratin (CK7) [2] and GATA-binding protein 3 (GATA3) [3] are two commonly used markers to confirm breast origin.

\footnotetext{
* Correspondence: slu@lifespan.org; ywang6@lifespan.org

Department of Pathology and Laboratory Medicine, Alpert Medical School of Brown University, 593 Eddy St; APC 12, Providence, RI 02903, USA
}

CK7 was first studied in breast tissue to differentiate luminal cells from myoepithelial cells [4]. Multiple subsequent studies have shown that CK7 was expressed in $89-98 \%$ of non-specified breast cancers [2, 5-8], in almost all medullary carcinomas [6], in the majority of micropapillary carcinoma of the breast [9] and in all mammary and extramammary Paget's disease [10]. CK7 was expressed in $97 \%$ of triple negative breast cancer with $14.5 \%$ demonstrating less than $20 \%$ tumor cell staining [11]. Its expression was lost in most sarcomatous ( $23 \%$ positivity) and fibromatosis-like ( $17 \%$ positivity) components, but was still retained in $71 \%$ of the matrix-producing component of metaplastic breast cancer [12].

(c) The Author(s). 2019 Open Access This article is distributed under the terms of the Creative Commons Attribution 4.0 International License (http://creativecommons.org/licenses/by/4.0/), which permits unrestricted use, distribution, and 
GATA3 belongs to the GATA family of zinc finger transcription factors and is involved in the development and morphogenesis of mammary glands [13]. GATA3 is considered a transcription factor maintaining the differentiation of luminal cells in the breast ducts [13]. It is one of the six genes (TP53, PIK3CA, AKT1, GATA3, CBFB and MAP3K1) with recurrent mutations in breast cancer [14]. GATA3 has been shown to be associated with the luminal subtype of breast cancer, whereas $88 \%$ of estrogen receptor (ER)-negative tumors retained GATA3 expression [15]. Its expression rate in triple-negative cancer ranged from 20.16 to $48 \%[16,17]$ in contrast to $74.6 \%$ in apocrine type triple-negative breast cancer [18]. The majority of published studies suggest that loss of GATA3 expression is associated with worse prognosis [19]; however, this is not universally accepted [3].

There are no systematic clinicopathological studies of CK7 and GATA3 negative tumors while limited studies are available characterizing the prognostic utility of GATA3 expression in breast cancer. In the current study, we analyzed 361 cases of breast cancers (196 cases of Nottingham Grade 3 breast cancers and 159 cases of Grade 1-2 cancers) to delineate the clinicopathologic features of CK7-negative and GATA3-negative tumors and their associations with patient outcome.

\section{Methods}

The study was performed in accordance with the ethical guidelines and approval from the Institutional Review Boards of Lifespan Health System (Rhode Island, United States).

\section{Patients}

All cases of primary breast cancer were retrieved from the pathology archive at the Lifespan Rhode Island and The Miriam Hospitals from 2000 to 2011. The study included 196 consecutive cases of Nottingham Grade 3 and 159 cases of Nottingham Grade 1 and Grade 2 for comparison. Clinicopathological data was collected from pathological reports and medical chart review. Survival data was acquired from the Lifespan Cancer Registry and medical chart review. Chemotherapy, hormonal treatment, or radiation was considered having chemo/radiation treatment.

\section{Tissue microarray construction and immunohistochemical} stain

Formalin-fixed paraffin-embedded tissue blocks with representative tumor areas were identified through review of corresponding hematoxylin and eosin-stained sections. Areas of interest were identified and marked on each selected block. The block was cored using a 1$\mathrm{mm}$ core needle. For each case four representative cores of tumor and 1 core of adjacent non-neoplastic tissue were arrayed. The cores were transferred to the recipient "master block" using a Beecher Tissue Microarrayer (Beecher Instruments, Silver Spring, MD).

Immunohistochemical staining was performed using the Dako Autostainer Plus and EnVision Dual Link detection reagent (DAKO; Carpinteria, CA) with DAB (Dako). The following primary antibodies were used: clone OV-TL12/3 against CK7 (ready to use; DAKO) and clone L50-823 against GATA3 (1:100 dilution; Biocare Medical, Pacheco, CA). ER and PR stains were considered positive if immunostaining was seen in more than $1 \%$ of tumor nuclei. Her2 immunostain score of $3 /$ 3 or confirmed Her2/neu gene amplification were considered HER2 positive. CK7 and GATA3 staining was considered positive if $1 \%$ or more of tumor cells were stained with at least mild intensity. Immunohistochemical stains were reviewed together by two pathologists (SL and YW) to minimize inter-observer variability. Information of Nottingham grade (Grade 1, Grade 2 or Grade 3) was obtained from pathology reports.

\section{Statistical methods}

$\mathrm{x} 2$ analysis was applied to evaluate associations between categorical variables. Fisher's test was used to replace $\mathrm{x} 2$ analysis when appropriate. T-test was used to compare between two continuous variables. For time-to-event measures, the Kaplan-Meier method was used to estimate the empirical survival and log-rank estimates were used. For Cox proportional hazard analysis, Wald test algorithm was used. All tests were 2-sided using a $p$-value of 0.05 as threshold for statistical significance. All analyses were performed using JMP Pro 14 (SAS, Cary, $\mathrm{NC})$. It was a large dataset of over 300 cases. In a small number of cases, part of clinical or staining information was not available. Minor discrepancies in case numbers were present; however, they did not affect percentages and conclusions.

\section{Results}

Clinicopathological features of CK7 negative and GATA3 negative breast cancers

Representative tumors with strong cytoplasmic staining of CK7 and nuclear staining of GATA3 are shown in Fig. 1. Thirteen percent (13\%) of Nottingham Grade 3 (G3) tumors were negative for CK7, while the negative rates were 9 and $2 \%$ for Grade 2 and Grade 1 tumors, respectively $(P=0.0457)$ (Table 1$)$. The negative rate for GATA3 was as high as $28 \%$ in Grade 3 tumors, while only 5 and $2 \%$ of Grade 2 and Grade 1 tumors were GATA3 negative $(P<0.0001)$ (Table 2$)$. After cases were stratified into two groups (Grade 1 and Grade 2 vs. Grade 3), patients with CK7 negative and GATA3 negative tumors had no age difference from those with CK7 and GATA3 positive tumors. No significant association 


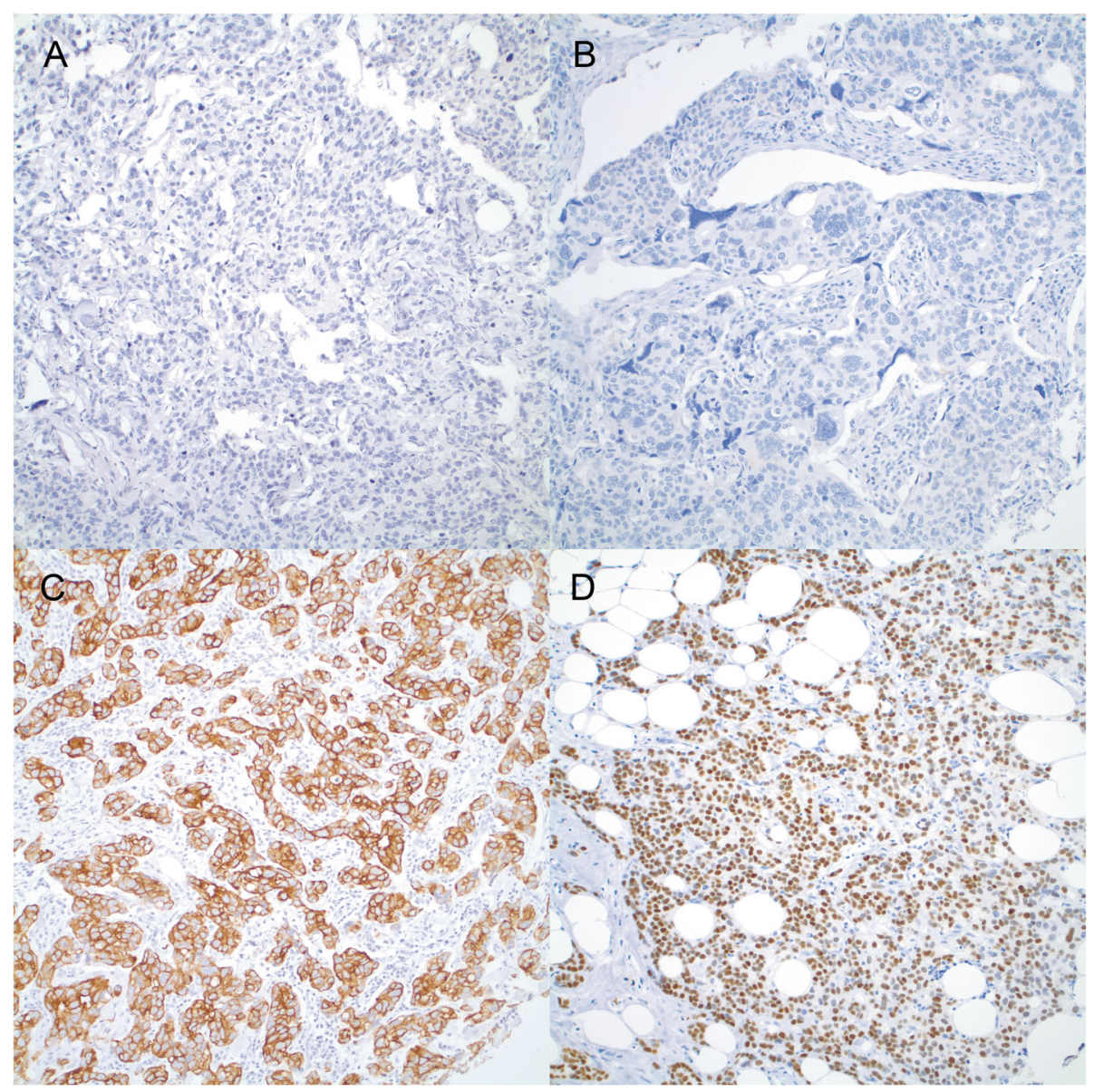

Fig. 1 Representative breast cancers of CK7 negative (a), GATA3 negative (b), CK7 positive (c), and GATA3 positive tumors (d)

was seen between CK7 and GATA3 expression, pTNM stages and Chemo/radiation treatment (Tables 1 and 2).

Next we analyzed CK7 and GATA3 expression among the different histologic types of breast cancer. The distribution of CK7 negative tumors in ductal cancers was not associated with tumor grade $(P=$ 0.7565), while more GATA3 negative tumors occurred in Grade 3 ductal cancers than those in Grade 1 and Grade 2 tumors, $27 \%$ vs. $6 \%(P<0.0001)$ (Table 3$)$. All lobular, mixed lobular and ductal, cribriform, micropapillary, mucinous, and tubular tumors in this series were CK7 and GATA3 positive (Table 3). All 10 metaplastic cancers were $\mathrm{G} 3$ and 30 and $70 \%$ of them were CK7 and GATA3 negative, respectively (Table 3). One apocrine cancer was positive for CK7 but negative for GATA3. (Table 3).

\section{Receptor status and molecular subtype in CK7 negative and GATA3 negative grade 3 breast cancers}

ER, PR, and Her2 expression were not found to be associated with CK7 expression in Grade 3 breast cancer $(P=$ $0.2822,0.0270$ and 0.9434 , respectively) (Table 3). About
$50 \%$ of ER negative Grade 3 tumors were also GATA3 negative, in contrast to $2 \%$ of ER positive tumors being GATA3 negative $(P<0.0001)$. Similarly, $42 \%$ of PR negative Grade 3 tumors were GATA3 negative, in contrast to $6 \%$ in PR positive tumors $(P<0.0001)$. More GATA3 negative tumors were seen in Her2 negative (31\%) than those in positive tumors (18\%); however, the difference was not statistically significant $(P=0.0826)$ (Table 3$)$.

Based on the expression of ER and HER2, Grade 3 tumors were grouped into luminal (ER+/HER2-), HER2 enriched (HER2+/ any ER), and basal like (ER-, HER2-) $[20,21]$. CK7 negative tumors were evenly distributed in all the subtypes $(P=0.6154)$, while GATA3 negative tumors were enriched in the basal-like group $(\mathrm{P}<0.0001)$ (Table 3$)$.

\section{Characteristics of CK7 and GATA3 double negative breast cancers}

There were 8 tumors negative for both CK7 and GATA3 (2.4\% of all), including 6 ductal and 2 metaplastic tumors. Seven out of $8(87.5 \%)$ were Grade 3 tumors; the remaining one was Grade 2. All 8 tumors were negative for both ER and PR. Only one was 
Table 1 Clinicopathological features of CK7 negative breast cancers

\begin{tabular}{|c|c|c|c|c|c|c|}
\hline \multirow{2}{*}{$\begin{array}{l}\text { Nottingham grade } \\
\text { Ck7 expression }\end{array}$} & \multicolumn{3}{|c|}{ Low-grade (Grade 1 and 2) } & \multicolumn{3}{|c|}{ High-grade (Grade 3) } \\
\hline & Negative & Positive & $P$-value & Negative & Positive & $P$-value \\
\hline $\operatorname{Age}^{a}(N)$ & $66 \pm 4.4(11)$ & $63.4 \pm 1.2(142)$ & 0.5615 & $62 \pm 3.3(25)$ & $61 \pm 1.2(168)$ & 0.7941 \\
\hline Nottingham grade & & & & & & 0.0457 \\
\hline 1 & $1(2 \%)$ & $45(98 \%)$ & & & & \\
\hline 2 & $10(9 \%)$ & 97 (91\%) & & & & \\
\hline 3 & & & & $25(13 \%)$ & $168(87 \%)$ & \\
\hline pT stage & & & 0.8569 & & & 0.3657 \\
\hline 1 & $8(7 \%)$ & $103(93 \%)$ & & $11(11 \%)$ & 87 (89\%) & \\
\hline 2 & $3(9 \%)$ & 30 (91\%) & & $9(13 \%)$ & $63(88 \%)$ & \\
\hline 3 & 0 & $5(100 \%)$ & & $2(14 \%)$ & $12(86 \%)$ & \\
\hline 4 & 0 & $4(100 \%)$ & & $2(33 \%)$ & $4(67 \%)$ & \\
\hline pN stage & & & 0.1377 & & & 0.7870 \\
\hline 0 & $8(9 \%)$ & $82(91 \%)$ & & $15(15 \%)$ & 85 (85\%) & \\
\hline 1 & 0 & 35 (100\%) & & $5(11 \%)$ & $42(89 \%)$ & \\
\hline 2 & 0 & $6(100 \%)$ & & $1(8 \%)$ & $12(92 \%)$ & \\
\hline 3 & $1(20 \%)$ & $4(80 \%)$ & & $1(20 \%)$ & $4(80 \%)$ & \\
\hline$x$ & $2(12 \%)$ & $15(88 \%)$ & & $2(8 \%)$ & $22(92 \%)$ & \\
\hline pM stage & & & 0.0738 & & & 0.4031 \\
\hline 0 & $6(6 \%)$ & $102(95 \%)$ & & $16(13 \%)$ & $106(87 \%)$ & \\
\hline 1 & 0 & 0 & & 0 & $2(100 \%)$ & \\
\hline$x$ & $4(17 \%)$ & $19(83 \%)$ & & $2(5 \%)$ & 37 (95\%) & \\
\hline Chemo/radiation therapy & & & 0.2462 & & & 0.4921 \\
\hline Yes & $6(6 \%)$ & 98 (94\%) & & $15(12 \%)$ & $115(88 \%)$ & \\
\hline No & $4(12 \%)$ & $29(88 \%)$ & & $7(16 \%)$ & $38(84 \%)$ & \\
\hline
\end{tabular}

${ }^{\mathrm{a} A g e}$ and tumor size: mean \pm SEM (standard error of mean)

positive for Her2 (Table 4). Among Grade 3 tumors, $17 \%$ of ER positive and 21\% PR positive tumors were seen in CK7 negative/GATA3 positive tumors, in contrast to $1 \%$ of ER positive and $45 \% \mathrm{PR}$ positive tumors being CK7 positive/GATA3 negative $(P<0.0001)$ (Table 4). Her2 expression did not have any association with CK7/GATA3 status $(P=0.4027)$ (Table 4$)$.

\section{Clinical outcomes in CK7 negative and GATA3 negative tumor patients}

The mean follow-up time in this series was 102 months with the longest of 216 months. Patients lost to followup or who died of causes other than breast cancer were censored. Patients with Grade 3 tumor were included in the survival analysis. Patients with CK7 negative tumors showed worse overall survival as compared to those with CK7 positive tumors; however, the difference was not statistically significant $(P=0.0890)$ (Fig. 2a). Patients with GATA3 negative tumors initially showed worse prognosis than these with GATA3 positive tumors, but the difference narrowed toward the end of the observation $(P=0.2320)$ (Fig. 2b). Grade 3 tumors in absence of both CK7 and GATA3 showed trend of worse patient outcome; however, there was no statistical significance $(P=0.2014)$.

In univariate Cox proportional hazard analyses, Her2, pT stage, and $\mathrm{pN}$ stage showed significant impacts on the prognosis of patients with Grade 3 tumors $(P=0.0018$, $P<0.0001, P=0.0012$, respectively) (Table 5). In multivariate analysis, ER, pT stage, and $\mathrm{pN}$ stage showed significant prognostic impact $(P=0.0314, P=0.0143$ and $P=0.0317$, respectively). CK7 and GATA3 did not show significant prognostic impact in both univariate and multivariate analyses (Table 5).

\section{Discussion}

Since there are no systematic clinicopathological studies on CK7 and GATA3 negative breast tumors and limited studies characterizing the prognostic utility of GATA3 expression in breast cancer, the current study provided detailed information that is lacking in the literature.

CK7 staining is not routinely used in the primary breast cancer diagnosis except in rare cases when clinical and pathological evidence prompt the need to 
Table 2 Clinicopathological features of GATA3 negative breast cancers

\begin{tabular}{|c|c|c|c|c|c|c|}
\hline \multirow{2}{*}{$\begin{array}{l}\text { Nottingham grade } \\
\text { Gata-3 expression }\end{array}$} & \multicolumn{3}{|c|}{ Low-grade (Grade 1 and Grade 2) } & \multicolumn{3}{|c|}{ High-grade (Grade 3) } \\
\hline & Negative & Positive & $P$-value & Negative & Positive & $P$-value \\
\hline $\operatorname{Age}^{a}(\mathrm{~N})$ & $69 \pm 6.7(7)$ & $64 \pm 1.1(152)$ & 0.3550 & $57 \pm 1.8(54)$ & $62 \pm 1.4(142)$ & 0.0732 \\
\hline Nottingham Grade & & & & & & $<0.0001$ \\
\hline 1 & $1(2 \%)$ & $47(98 \%)$ & & & & \\
\hline 2 & $6(5 \%)$ & $105(95 \%)$ & & & & \\
\hline 3 & & & & $54(28 \%)$ & $142(72 \%)$ & \\
\hline pT stage & & & 0.4673 & & & 0.2223 \\
\hline 1 & $4(3 \%)$ & $112(97 \%)$ & & $31(32 \%)$ & $67(69 \%)$ & \\
\hline 2 & $3(9 \%)$ & $31(91 \%)$ & & $15(21 \%)$ & $58(79 \%)$ & \\
\hline 3 & 0 & $5(100 \%)$ & & $6(40 \%)$ & $9(60 \%)$ & \\
\hline 4 & 0 & $4(100 \%)$ & & $1(14 \%)$ & $6(86 \%)$ & \\
\hline pN stage & & & 0.4247 & & & 0.2580 \\
\hline 0 & $3(3 \%)$ & $93(97 \%)$ & & $32(32 \%)$ & $66(67 \%)$ & \\
\hline 1 & $1(3 \%)$ & $33(97 \%)$ & & $10(20 \%)$ & $40(80 \%)$ & \\
\hline 2 & $1(17 \%)$ & $5(83 \%)$ & & $2(17 \%)$ & $10(83 \%)$ & \\
\hline 3 & 0 & $5(100 \%)$ & & 0 & $5(100 \%)$ & \\
\hline$x$ & $2(11 \%)$ & $16(89 \%)$ & & $9(33 \%)$ & $18(67 \%)$ & \\
\hline pM stage & & & 0.6172 & & & 0.9294 \\
\hline 0 & $5(5 \%)$ & $106(95 \%)$ & & $36(28 \%)$ & 91 (72\%) & \\
\hline 1 & 0 & 0 & & $1(33 \%)$ & $2(67 \%)$ & \\
\hline$x$ & $2(8 \%)$ & $24(92 \%)$ & & $12(31 \%)$ & $51(69 \%)$ & \\
\hline Chemo/radiation Therapy & & & 0.2952 & & & 0.2785 \\
\hline Yes & $4(4 \%)$ & $103(96 \%)$ & & $39(29 \%)$ & 94 (71\%) & \\
\hline No & $3(8 \%)$ & 33 (91\%) & & $10(21 \%)$ & 37 (79\%) & \\
\hline
\end{tabular}

${ }^{a}$ Age: mean \pm SEM (standard error of mean)

exclude a secondary cancer source. CK7 negative breast tumors may be diagnostically challenging in metastatic tumors and cancers of unknown origin. A comprehensive characterization of CK7 negative breast cancer may be scientifically and diagnostically useful.

In the current study, we found that as high as $13 \%$ of Grade 3 breast cancers and 30\% of metaplastic cancers were CK7 negative. CK7 expression was not significantly associated with age, stage, receptor status and molecular subtype. Among the 11 histologic types included in the study, loss of CK7 expression was only seen in ductal or metaplastic tumors.

Based on Grade 3 breast cancers, survival analysis showed CK7 expression had no impact on overall outcomes (Fig. 2). Given the fact that Grade 1 and 2 tumors are more likely to retain CK7 expression and associated with better prognoses, survival analysis of breast tumors of expected grade distribution (about 34\% of Grade 3 instead of $55 \%$ in this series, see below) will likely show significant association of CK7 expression loss with poor patient outcome. Indeed, if Grade 1 and Grade 2 tumors were included in the current series, a significant association were seen between worse overall survival and CK7 expression loss patients $(P=0.0084)$ (Additional file 1 : Figure S1A). Loss of CK7 in the tumor could indicate initiation of a different differentiation pathway, presence of epithelial to mesenchymal transition, dedifferentiation, or enhanced tumor "stemness". More studies should be carried out for further investigations.

As opposed to CK7 negative tumors, GATA3 negative breast cancer has gained more attention from both diagnostic pathologists and cancer biologists. The percentage of GATA3 negative breast cancers vary from study to study, ranging from 0 to $46 \%$ in ER positive tumors and from 17 to $97 \%$ in estrogen negative cancers [3]. In the current study, the negative rates were $1.7 \%$ in ER positive and $48.6 \%$ in ER negative tumors. Among the published studies in which the same antibody and the same positivity cut-off were used as in this study, GATA3 negative rates ranged from 17 to $56 \%$, consistent with our finding of $17.1 \%$. Reported GATA3 negative rates in metaplastic cancers ranged from 44 to $82 \%$, which was consistent with the $70 \%$ (7 out of 10) in the current 
Table 3 Histologic types and molecular features of CK7 negative and GATA3 negative breast cancers

\begin{tabular}{|c|c|c|c|c|c|c|}
\hline Histologic type and molecular features & CK7 negative & CK7 positive & $P$-value & GATA3 negative & GATA3 positive & $P$-value \\
\hline Ductal & & & 0.7565 & & & $<0.001$ \\
\hline Grade 3 & $22(13 \%)$ & 147 (87\%) & & 47 (27\%) & $126(73 \%)$ & \\
\hline Grade 1 \& Grade 2 & $11(12 \%)$ & $83(88 \%)$ & & $6(6 \%)$ & $91(94 \%)$ & \\
\hline \multicolumn{7}{|l|}{ Lobular \& Mixed } \\
\hline Grade 3 & 0 & 37 & & 0 & 36 & \\
\hline Grade 1-Grade 2 & 0 & 18 & & 0 & 20 & \\
\hline Metaplastic (all Grade 3) & $3(30 \%)$ & $7(70 \%)$ & & $7(70 \%)$ & $3(30 \%)$ & \\
\hline Cribriform (all Grade 1-Grade 2) & 0 & 5 & & 0 & 6 & \\
\hline Micropapillary (all Grade 1-Grade 2) & 0 & 4 & & 0 & 4 & \\
\hline Mucinous (all Grade 1-Grade 2) & 0 & 5 & & 0 & 5 & \\
\hline Tubular (all Grade 1-Grade 2) & 0 & 3 & & 0 & 3 & \\
\hline Apocrine (Grade 2) & 0 & 1 & & 1 & 0 & \\
\hline ER (Grade 3 only) & & & 0.2822 & & & $<0.0001$ \\
\hline Positive & $14(16 \%)$ & 72 (84\%) & & $2(2 \%)$ & 89 (98\%) & \\
\hline Negative & $11(10 \%)$ & 95 (90\%) & & $52(50 \%)$ & $52(50 \%)$ & \\
\hline PR (Grade 3 only) & & & 0.0270 & & & $<0.0001$ \\
\hline Positive & $15(20 \%)$ & $61(80 \%)$ & & $5(6 \%)$ & 74 (94\%) & \\
\hline Negative & $10(9 \%)$ & 106 (91\%) & & 49 (42\%) & $67(58 \%)$ & \\
\hline Her2 (Grade 3 only) & & & 0.9434 & & & 0.0826 \\
\hline Positive & $6(13 \%)$ & 39 (87\%) & & $9(18 \%)$ & 40 (82\%) & \\
\hline Negative & $19(13 \%)$ & $128(87 \%)$ & & 45 (31\%) & 101 (69\%) & \\
\hline Subtype (Grade 3 only) & & & 0.6154 & & & $<0.0001$ \\
\hline Luminal & $10(16 \%)$ & 76 (89\%) & & $1(2 \%)$ & $63(98 \%)$ & \\
\hline Her2 & $6(13 \%)$ & 39 (87\%) & & $9(18 \%)$ & 40 (82\%) & \\
\hline Basal-like & $9(11 \%)$ & 76 (90\%) & & 44 (54\%) & $38(46 \%)$ & \\
\hline
\end{tabular}

Table 4 Characteristics of CK7 and GATA3 double negative Grade 3 breast cancers

\begin{tabular}{|c|c|c|c|c|c|}
\hline Features & CK7-/GATA3- & CK7-/GATA3+ & CK7+/GATA3- & CK7+/GATA3+ & $P$-value \\
\hline Counts & $8(2.4 \%)$ & $28(85.4 \%)$ & $51(15.5 \%)$ & $241(73.5 \%)$ & \\
\hline Nottingham Grade & & & & & $<0.0001$ \\
\hline 1 & 0 & $1(2.3 \%)$ & $1(2.3 \%)$ & $41(95.3 \%)$ & \\
\hline 2 & $1(1 \%)$ & 9 (8.8\%) & $5(5 \%)$ & 87 (85.3\%) & \\
\hline 3 & 7 (3.8\%) & $18(9.8 \%)$ & 45 (24.5\%) & $113(61.7 \%)$ & \\
\hline ER (G3 only) & & & & & $<0.0001$ \\
\hline Positive & 0 & $14(17 \%)$ & $1(1 \%)$ & $69(82 \%)$ & \\
\hline Negative & $7(7 \%)$ & $4(4 \%)$ & $44(45 \%)$ & $43(43 \%)$ & \\
\hline $\mathrm{PR}(\mathrm{HG})$ & & & & & $<0.0001$ \\
\hline Positive & 0 & $15(21 \%)$ & $4(5 \%)$ & $54(74 \%)$ & \\
\hline Negative & $7(6 \%)$ & $3(3 \%)$ & $41(38 \%)$ & $58(53 \%)$ & \\
\hline Her2 (HG) & & & & & 0.4027 \\
\hline Positive & $1(2 \%)$ & $5(12 \%)$ & $7(16 \%)$ & $30(70 \%)$ & \\
\hline Negative & $6(4 \%)$ & $13(9 \%)$ & $38(27 \%)$ & 92 (59\%) & \\
\hline
\end{tabular}



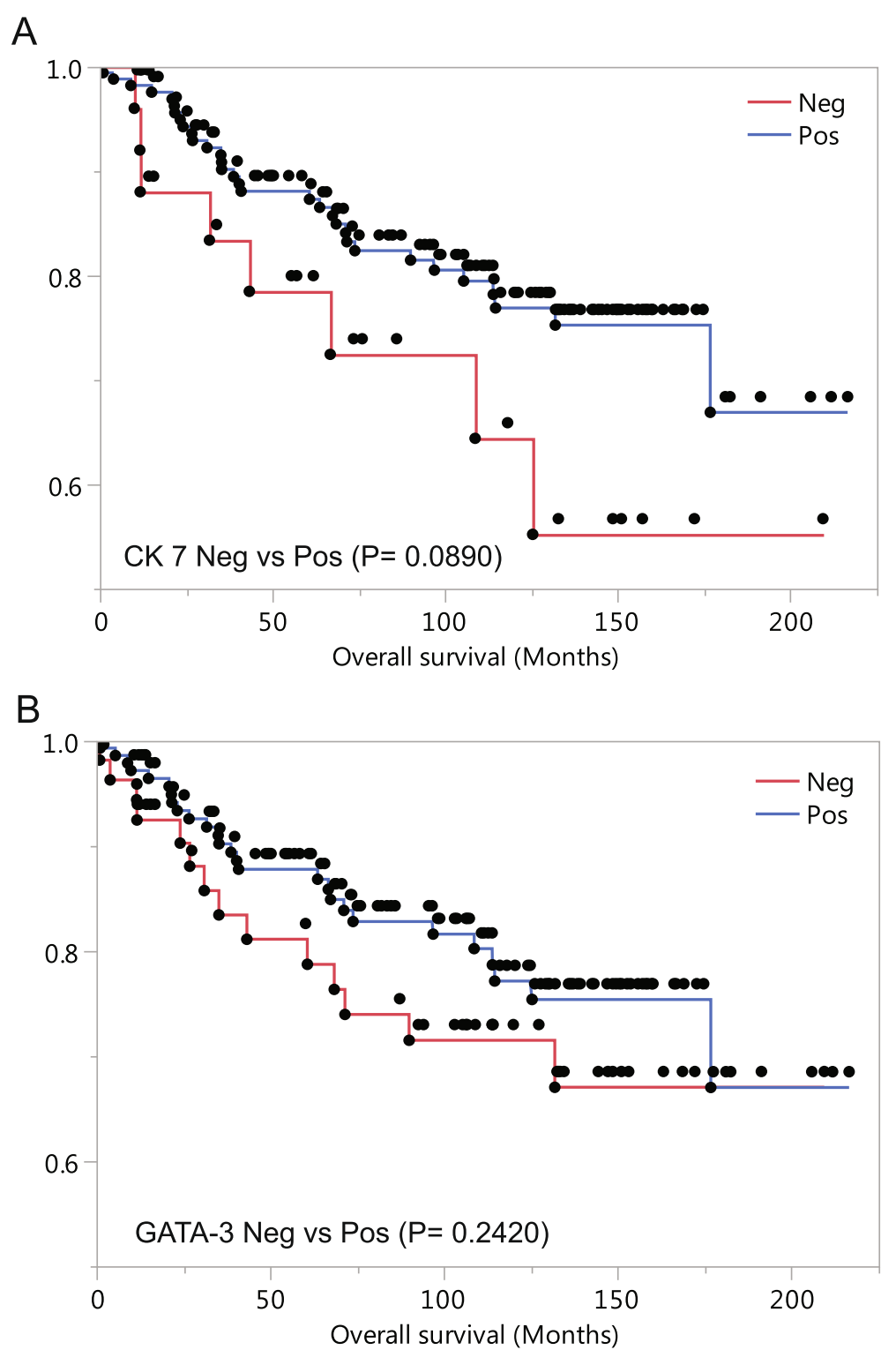

Fig. 2 Survival analysis of CK7 negative and GATA3 negative Grade 3 breast cancer patients. a Overall survival in CK7 negative vs. positive cancer patients. b Overall survival in GATA3 negative vs. positive cancer patients

study. In addition we found that none of the lobular tumors and those with mixed lobular and ductal features was GATA3 negative, which was compatible with most published studies [3].

GATA3 is required for ER-dependent cellular processes and GATA3 and ER participate in positive feedback loops, each stimulating the expression of the other [21]. Forced expression of GATA3 in mouse mammary cancer model showed a protective effect with improved prognosis [22]. Mutations of GATA3 in breast cancer are relatively common. Based on analyses performed through cBioPortal $[23,24]$ on the largest publicly available breast cancer dataset, Molecular Taxonomy of Breast Cancer International
Consortium (METABRIC) [25], 11.5\% (250/2173) of breast tumors harbored somatic mutations of GATA3. Out of the 250 mutations, 193 (77.2\%) were truncating, 52 (20.8\%) were missense and 5 (2\%) were inframe mutations. GATA3 mutations were more frequently seen in mixed invasive and mucinous cancers $(P=0.0124)$ and more likely to be estrogen receptor positive $(P<0.0001)$, Her2 negative $(P=0.0002)$, with lower grades $(P<0.0001)$, and with lower $\mathrm{pT}$ stages (Grade 1 and 2) $(P=0.0254)$ (Additional file 1: Table S1). GATA3 mutated tumors were associated with better patient outcomes $(P=0.0018)$ (Additional file1: Figure S2). Mutant GATA3 proteins mainly interfere with the DNA binding but the transactivation domains are largely intact [22]. GATA3 
Table 5 Univariate and multi-variants Cox proportional hazard analyses of CK7 and Gata3 expression in Grade 3 breast cancers

\begin{tabular}{|c|c|c|c|c|}
\hline Parameter & Univariate [lower 95\%, upper 95\%] & $P$-value & Multi-variant [lower 95\%, upper 95\%] & $P$-value \\
\hline Age & {$[-0.025386,0.015766]$} & 0.6547 & {$[-0.062527,0.0038646]$} & 0.0858 \\
\hline CK7 [Pos-Neg] & {$[-1.386215,0.1862815]$} & 0.0949 & {$[-1.512252,0.4173403]$} & 0.1972 \\
\hline Gata3 [Pos-Neg] & {$[-1.013242,0.2908032]$} & 0.2556 & {$[-0.969737,1.0802151]$} & 0.9156 \\
\hline ER [Pos-Neg] & {$[-1.1454,0.0346665]$} & 0.0659 & {$[-2.069892,-0.1064]$} & 0.0314 \\
\hline Her2 [Pos-Neg] & {$[-1.858073,-0.233523]$} & 0.0081 & {$[-2.013318,0.0923009]$} & 0.1037 \\
\hline pT & & $<0.0001$ & & 0.0143 \\
\hline $\mathrm{T} 2-\mathrm{T} 1$ & {$[-0.191511,1.129611]$} & & {$[-0.643541,1.1878657]$} & \\
\hline $\mathrm{T} 3-\mathrm{T} 2$ & {$[0.6830868,2.4074455]$} & & {$[-0.127867,2.283623]$} & \\
\hline T4-T3 & {$[-1.232561,1.0674436]$} & & {$[-1.01393,2.770524]$} & \\
\hline $\mathrm{pN}$ & & 0.0012 & & 0.0317 \\
\hline N1-N0 & {$[-0.025245,1.3477474]$} & & {$[0.1051354,1.9312744]$} & \\
\hline N2-N1 & {$[-0.767612,1.439705]$} & & {$[-3.543852,1.1203884]$} & \\
\hline N3-N2 & {$[0.0520156,2.7110952]$} & & {$[-0.497292,4.7129041]$} & \\
\hline $\mathrm{Nx}-\mathrm{N} 3$ & {$[-2.562949,-0.230165]$} & & {$[-2.618863,0.859423]$} & \\
\hline Chemo/radiation & {$[-0.742833,0.6887183]$} & 0.8420 & {$[-1.389767,0.830584]$} & 0.5446 \\
\hline
\end{tabular}

antibody we used (clone L50-823) was raised against peptide segment between transactivation and DNA-binding domains and presumably captures both wild-type and mutant protein versions. In the current study, GATA3 was positive in half of the ER negative tumors, consisting of $28 \%$ of all Grade 3 tumors (Table 3). Further investigations should be focused on the mechanisms how GATA3 maintains its expression without activating ER.

Published data regarding GATA3 as a prognostic marker are conflicting. Loss of GATA3 expression has been associated with unfavorable clinical outcome and worse survival $[15,26,27]$. However, no association with outcome has been observed in other studies [28]. In one study, GATA3 expression was found to be associated with favorable outcome in all the breast tumors in the study, while the association was lost when only ER positive cancers were analyzed [29]. GATA3 expression is closely associated with ER and PR expression and loss of GATA3 expression is postulated to be similar to loss of ER expression prognostically. However, our study did not see similar findings in 196 Grade 3 breast cancers. Multiple factors could contribute. First, the case number was not large enough to bear sufficient statistical power. Second, ER and GATA3 are closely related in the hormonal pathway, but they could still have distinct functions as to tumor progression. Third, in the current practice, vast majority of ER positive tumors have been treated hormonally. The treatment may impact the prognosis. Four, our survival study only included Grade 3 tumors and the adding more Grade 1 and Grade 2 tumors is likely to include more GATA3 positive tumors with better prognosis and thus with a significant prognostic difference. Indeed, repeating analysis by adding Grade 1 and Grade 2 tumors in the current series showed significant worse survival of GATA3 negative cancer patients $(P=0.0063)$ (Additional file 1: Figure S1B).

Our study is limited by a high proportion of Grade 3 breast cancer (55\%) while it was estimated that only a third of breast cancer are Grade 3 [30]. A significant amount of additional work would be involved if all the concurrent Grade 1 and Grade 2 tumors were included in the study. Results that would be affected by disproportional Grade 1 and Grade 2 cases include survival analyses and association studies when markers were not evenly distributed among grades, such as ER, PR, Her2 and pTNM status. Therefore, these analyses were performed only in Grade 3 tumors except association studies that directly involved the grade.

Another possible limitation is about the proportions of each special type cancer in this study. Our study included 10 metaplastic ( $0.28 \%$ of all tumors), 6 cribriform (1.6\%), 4 micropapillary (1.1\%), 5 mucinous (1.4\%), 3 tubular $(0.8 \%)$ and 1 apocrine cancer $(0.3 \%)$. The cribriform and tubular tumors were slightly overrepresented and mucinous and apocrine tumors were slightly underrepresented, comparing to known percentages for each special type: 0.2 to $5 \%$ for metaplastic, $0.3-0.8 \%$ for cribriform, $0.9-2 \%$ for micropapillary, $2 \%$ for mucinous, $2 \%$ for tubular and $4 \%$ for apocrine cancers [31]. However, the differences do not significantly affect the main conclusions in this study.

The mainly purpose of the study is to understand the pathobiology of the CK7 negative and GATA3 negative breast cancer. If we want to apply the findings in this study to facilitate the diagnosis of metastatic breast cancer, we need to know the CK7 and GATA3 expression 
in matched pairs of primary and metastasis. It certainly requires a separate study. A small portion of CK7 and/or GATA3 negative cases in our series later developed tumor metastasis; however, CK7 and GATA3 immunostains were not used to form the diagnoses of these metastases. Some of the metastases occurred before GATA3 immunostain became available. However, clinical information, microscopic morphology and other mammary gland markers were often enough to establish the diagnoses. In a pilot study, we collected 12 pairs of primary tumor and liver metastasis and 12 pairs of primary tumor and bone metastasis. There was no GATA3 expression change in primaries and liver metastases: all 10 GATA3 positive primaries retained GATA3 expression in their paired liver metastases and GATA3 expression in 2 negative primaries remained negative in the paired liver metastases. Some of the GATA3 expression was lost in bone metastasis: GATA3 expression in 8 positive primaries was only retained in 4 paired bone metastases and GATA3 expression in 4 negative primaries remained negative in the paired bone metastases. The exact reason why GATA3 expression was lost in some bone metastases is unclear. It could be true loss of expression (such as post-chemotherapy) but it is more likely to be related with tissue processing such as decalcification or sampling error due to the small size of bone biopsy. The same type of sampling error could happen to core biopsy or fine needle aspiration (FNA). Further studies are needed to explore GATA3 and CK7's role in diagnosing metastases of special cancer types, such as metaplastic, mucinous, and apocrine tumors.

In the current series, we identified 8 cases of CK7 and GATA3 double negative breast cancer. All eight cases have been considered as primary breast cancer and pathological diagnoses were made without CK7 and/or GATA3 stains. It could have been challenging if CK7 and GATA3 immunostains were performed at the time of diagnosis. During the current study, we had retrospective chart review for these cases including any follow-up history and no non-breast primary tumor was identified. In our practice, GATA3 immunostain is routinely performed on any triple-negative cancers that do not have an in situ component. Ultimately, only history and follow-up can definitively confirm the breast origin in some cases.

\section{Conclusions}

This is the first study that comprehensively characterized clinicopathological and prognostic features of breast cancers missing either of two canonical markers, CK7 and GATA3. There was 13\% CK7 negative and 28\% GATA3 negative tumors in Grade 3 breast cancer. CK7 negative tumors showed no association with most clinicopathological features and loss of CK7 expression was not associated with worse outcome in patients with Grade 3 tumors. Loss of GATA3 expression was associated with negative ER status and GATA3 negative tumors were enriched in the basal-like molecular subgroup. About half of the ER negative breast cancers retained GATA3 expression and no overall survival was seen associated with GATA3 negative Grade 3 tumors. Profiling the CK7 negative and GATA3 negative breast cancers helps to understand the biology of these specific tumor subgroups and may aid in their diagnosis.

\section{Supplementary information}

Supplementary information accompanies this paper at https://doi.org/10. 1186/s12885-019-6295-8.

Additional file 1: Table S1. Clinicopathological features of GATA3 mutated breast cancer in METABRIC dataset. Figure S1. Survival analysis of CK7 negative and GATA3 negative cancer patients (including Grade 13). A. Overall survival in CK7 negative vs. positive cancer patients. B. Overall survival in GATA3 negative vs. positive cancer patients. Figure S2. Survival analysis of 2173 patients/samples in the METABRIC studies based on GATA3 mutation status.

\section{Acknowledgements}

Not applicable.

\section{Authors' contributions}

SL and YW conceived the project and scored the immunostains. SL performed data analysis and prepared the manuscript. EY, LJW, MBR and YW helped in the project design and response to the comments. All authors read and approved the final manuscript.

\section{Funding}

Research funding for this study was provided by the Molecular Pathology Core of the COBRE Center for Cancer Research Development funded by the National Institute of General Medical Sciences of the National Institutes of Health under award number P20GM103421 (United States). The funding bodies have no role in study design, collection, analysis, interpretation of data or writing the manuscript.

\section{Availability of data and materials}

Deidentified patient data from this study will not be shared publicly, but are available from the corresponding author on reasonable request.

\section{Ethics approval and consent to participate}

An Institutional Review Board from Rhode Island Hospital has approved the study. The human materials were obtained from archived paraffin blocks that were remnants of what were used for pathologic diagnosis. Informed consent was not required by Institutional Review Board.

Consent for publication

No applicable.

\section{Competing interests}

Author SL declares that he has no competing interest. Author EY declares that he has no competing interest. Author LJW declares that she has no competing interest. Author MBR declares that he has no competing interest. Author YW declares that he has no competing interest.

Received: 11 July 2019 Accepted: 25 October 2019

Published online: 12 November 2019

\section{References}

1. Siegel RL, Miller KD, Jemal A. Cancer statistics, 2018. CA Cancer J Clin. 2018;68:7-30. 
2. Shao MM, Chan SK, Yu AM, Lam CC, Tsang JY, Lui PC, et al. Keratin expression in breast cancers. Virchows Arch. 2012;461(3):313-22.

3. Asch-Kendrick R, Cimino-Mathews A. The role of GATA3 in breast carcinomas: a review. Hum Pathol. 2016:48:37-47.

4. Altmannsberger M, Dirk T, Droese M, Weber K, Osborn M. Keratin polypeptide distribution in benign and malignant breast tumors: subdivision of ductal carcinomas using monoclonal antibodies. Virchows Arch B Cell Pathol Incl Mol Pathol. 1986;51(3):265-75.

5. Malzahn K, Mitze M, Thoenes M, Moll R. Biological and prognostic significance of stratified epithelial cytokeratins in infiltrating ductal breast carcinomas. Virchows Arch. 1998:433:119-29.

6. Tot T. Patterns of distribution of cytokeratins 20 and 7 in special types of invasive breast carcinoma: a study of 123 cases. Ann Diagn Pathol. 1999;3(6):350-6.

7. Ramaekers F, Van Niekerk C, Poels L, et al. Use of monoclonal antibodies to keratin 7 in the differential diagno Tot T. the cytokeratin profile of medullary carcinoma of the breast. Histopathology. 2000;37(2):175-81.

8. Gloyeske NC, Woodard AH, Elishaev E, Yu J, Clark BZ, Dabbs DJ, et al. Immunohistochemical profile of breast cancer with respect to ER and HER2 status. Appl Immunohistochem Mol Morphol. 2015;23(3):202-8.

9. Kim MJ, Gong G, Joo HJ, Ahn SH, Ro JY. Immunohistochemical and clinicopathologic characteristics of invasive ductal carcinoma of breast with micropapillary carcinoma component. Arch Pathol Lab Med. 2005; 129(10):1277-82.

10. Liegl B, Leibl S, Gogg-Kamerer M, Tessaro B, Horn LC, Moinfar F. Mammary and extramammary Paget's disease: an immunohistochemical study of 83 cases. Histopathology. 2007;50(4):439-47.

11. Davion SM, Siziopikou KP, Sullivan ME. Cytokeratin 7: a re-evaluation of the 'tried and true' in triple-negative breast cancers. Histopathology. 2012;61 (4):660-6.

12. Rakha EA, Coimbra ND, Hodi Z, Juneinah E, Ellis IO, Lee AH. Immunoprofile of metaplastic carcinomas of the breast. Histopathology. 2017;70(6):975-85.

13. Kouros-Mehr H, Slorach EM, Sternlicht MD, Werb Z. GATA3 maintains the differentiation of the luminal cell fate in the mammary gland. Cell. 2006; 127(5):1041-55.

14. Banerji S, Cibulskis K, Rangel-Escareno C, Brown KK, Carter SL, Frederick AM, et al. Sequence analysis of mutations and translocations across breast cancer subtypes. Nature. 2012;486(7403):405-9.

15. McCleskey BC, Penedo TL, Zhang K, Hameed O, Siegal GP, Wei S. GATA3 expression in advanced breast cancer: prognostic value and organ-specific relapse. Am J Clin Pathol. 2015;144(5):756-63.

16. Shaoxian T, Baohua Y, Xiaoli X, Yufan C, Xiaoyu T, Hongfen L, et al. Characterisation of GATA3 expression in invasive breast cancer: differences in histological subtypes and immunohistochemically defined molecular subtypes. J Clin Pathol. 2017;70(11):926-34.

17. Byrne DJ, Deb S, Takano EA, Fox SB. GATA3 expression in triple-negative breast cancers. Histopathology. 2017;71(1):63-71.

18. Kim S, Moon BI, Lim W, Park S, Cho MS, Sung SH. Expression patterns of GATA3 and the androgen receptor are strongly correlated in patients with triple-negative breast cancer. Hum Pathol. 2016;55:190-5. https://doi.org/10. 1016/j.humpath.2016.04.013 Epub 2016 May 13. PubMed PMID: 27184484.

19. Guo Y, Yu P, Liu Z, Maimaiti Y, Chen C, Zhang Y, et al. Prognostic and clinicopathological value of GATA binding protein 3 in breast cancer: a systematic review and meta-analysis. PLoS One. 2017;12(4):e0174843. https://doi.org/10.1371/journal.pone.0174843 eCollection 2017. Review. PubMed PMID: 28394898; PubMed Central PMCID: PMC5386271.

20. TO N, Hsu FD, Jensen $\mathrm{K}$, et al. Immunohistochemical and clinical characterization of the basal-like subtype of invasive breast carcinoma. Clin Cancer Res. 2004;10:5367-74.

21. Eeckhoute J, Keeton EK, Lupien M, Krum SA, Carroll JS, Brown M. Positive cross-regulatory loop ties GATA-3 to estrogen receptor alpha expression in breast cancer. Cancer Res. 2007;67(13):6477-83.

22. Takaku M, Grimm SA, Wade PA. GATA3 in breast cancer: tumor suppressor or oncogene? Gene Expr. 2015;16(4):163-8.

23. Cerami E, Gao J, Dogrusoz U, Gross BE, Sumer SO, Aksoy BA, et al. The cBio cancer genomics portal: an open platform for exploring multidimensional cancer genomics data. Cancer Discov. 2012;2(5):401-4.

24. Gao J, Aksoy BA, Dogrusoz U, Dresdner G, Gross B, Sumer SO, et al. Integrative analysis of complex cancer genomics and clinical profiles using the cBioPortal. Sci Signal. 2013;6(269):11.

25. Curtis C, Shah SP, Chin SF, Turashvili G, Rueda OM, Dunning MJ, et al. McKinney S; METABRIC group, Langerød a, green a, Provenzano E, Wishart G, Pinder S, Watson P, Markowetz F, murphy L, Ellis I, Purushotham a,
Børresen-dale AL, Brenton JD, Tavaré S, Caldas C, Aparicio S. the genomic and transcriptomic architecture of 2,000 breast tumours reveals novel subgroups. Nature. 2012;486(7403):346-52.

26. Mehra R, Varambally S, Ding L, Shen R, Sabel MS, Ghosh D, et al. Identification of GATA3 as a breast cancer prognostic marker by global gene expression meta-analysis. Cancer Res. 2005;65(24):11259-64.

27. Yoon NK, Maresh EL, Shen D, Elshimali Y, Apple S, Horvath S, et al. Higher levels of GATA3 predict better survival in women with breast cancer. Hum Pathol. 2010;41(12):1794-801.

28. Ni YB, Tsang JY, Chan SK, Tse GM. GATA-binding protein 3, gross cystic disease fluid protein-15 and mammaglobin have distinct prognostic implications in different invasive breast carcinoma subgroups. Histopathology. 2015;67(1):96-105.

29. Voduc D, Cheang M, Nielsen T. GATA-3 expression in breast cancer has a strong association with estrogen receptor but lacks independent prognostic value. Cancer Epidemiol Biomark Prev. 2008;17(2):365-73.

30. Rakha EA, Reis-Filho JS, Baehner F, Dabbs DJ, Decker T, Eusebi V, et al. Breast cancer prognostic classification in the molecular era: the role of histological grade. Breast Cancer Res. 2010;12(4):207.

31. Lakhani SR, Ellis IO, Schnitt SJ, Tan PH, van de Vijver MJ, editors. WHO classification of tumors of the breast. Lyon: IARC Press; 2012.

\section{Publisher's Note}

Springer Nature remains neutral with regard to jurisdictional claims in published maps and institutional affiliations.
Ready to submit your research? Choose BMC and benefit from:

- fast, convenient online submission

- thorough peer review by experienced researchers in your field

- rapid publication on acceptance

- support for research data, including large and complex data types

- gold Open Access which fosters wider collaboration and increased citations

- maximum visibility for your research: over $100 \mathrm{M}$ website views per year

At BMC, research is always in progress.

Learn more biomedcentral.com/submissions 